Bull. Austral. Math. Soc.

VoL. 53 (1996) [197-207]

\title{
THE EXISTENCE OF BOUNDED HARMONIC FUNCTIONS ON C-H MANIFOLDS
}

\section{Qing Ding ANd Detang Zhou}

Let $M$ be a Cartan-Hadamard manifold of dimension $n(n \geqslant 2)$. Suppose that $M$ satisfies for every $x \in M$ outside a compact set an inequality:

$$
-b^{2} \leqslant \text { sectional curvature }(x) \leqslant-\frac{A}{\rho^{2}(x)}
$$

where $b, A$ are positive constants and $A>4$. Then $M$ admits a wealth of bounded harmonic functions, more precisely, the Dirichlet problem of the Laplacian of $M$ at infinity can be solved for any continuous boundary data on $S^{n-1}(\infty)$.

\section{INTRODUCTION}

The study of the existence or nonexistence of harmonic functions has led to new insights into the geometry of complete Riemannian manifolds. Yau [9] proved that on any complete noncompact Riemannian manifold $M$ there are no globally defined nontrivial harmonic functions in $L^{p}(M)$ for any $1<p<\infty$. In the case $p=\infty$, that is, for bounded harmonic functions, the conclusion is strongly influenced by the sign of the curvature of the manifolds. Yau [10] proved that there are no nontrivial bounded harmonic functions on manifolds of nonnegative Ricci curvature. Since then, the existence or nonexistence of nontrivial bounded harmonic functions on complete noncompact manifolds of nonpositive curvature has become an attractive topic both in analysis and geometry.

On one hand, Greene and Wu (Theorem D in [6]) proved that if $M$ is a complete noncompact Riemannian manifold satisfing:

$$
0 \geqslant \text { sectional curvature }(x) \geqslant-k(\rho(x))
$$

where $\rho(x)$ denotes the distance function from $x$ to a fixed point $O \in M, k$ is a nonnegative continuous function on $[0, \infty)$ such that $\int_{0}^{\infty} s k(s) d s<\infty$, then the exponential mappings of such a manifold are always quasi-isometries and every positive harmonic function (hence every bounded harmonic function) on $M$ is constant. On the other hand, there is a conjecture (see [6, Section 0]) as follows

\section{Received 27th April, 1995}

Supported partially by NNSFC and NSF of Shandong. The authors wish to thank Professors Chaohao $\mathrm{Gu}$ and Hesheng $\mathrm{Hu}$ for their encouragement and guidance.

Copyright Clearance Centre, Inc. Serial-fee code: 0004-9729/96 \$A2.00+0.00. 
Conjecture. If $M$ is a Cartan-Hadamard manifold which satisfies for every $x \in M$ an inequality outside a compact set:

$$
\text { sectional curvature }(x) \leqslant-\frac{A}{\rho^{2}(x)}
$$

where $\rho(x)$ is the distance function, $A$ is a positive constant, then $M$ possesses enough bounded harmonic functions.

A study along these lines was first done by Anderson [1] and Sullivan [8] independently in 1983. They proved that if the sectional curvature $K_{M}$ of $M$ satisfies $-b^{2} \leqslant K_{M} \leqslant-a^{2}$ for some constant $a, b>0$, then $M$ has a wealth of bounded harmonic functions, precisely, there is a geometric compactification of $M$ obtained by adding a sphere $S^{n-1}(\infty)$ at infinity and the Dirichlet problem of the Laplacian of $M$ can be solved for continuous boundary data on $S^{n-1}(\infty)$. In 1985, Anderson and Schoen studied in [2] the intersection between the geometry of such a manifold and some aspects of function theory on this space. Recently, the first author obtained in [5] an existence theorem for bounded harmonic functions on Cartan-Hadamard manifolds in terms of negative Ricci curvature pinching conditions and a relation of the angle and its sides, which gives Anderson or Sullivan's result as a special case. It should be mentioned that Ballmann [3] got a existence theorem for bounded harmonic functions for (symmetric) manifolds of rank 1 with nonpositive sectional curvature by using stochastic methods.

In this paper, we shall give a new existence theorem for bounded harmonic functions on Cartan-Hadamard manifolds, which provides a partial answer to the above conjecture. Namely, we shall establish

Theorem 1. Let $M$ be a Cartan-Hadamard manifold of dimension $n(n \geqslant 3)$. Suppose that $M$ satisfies for every $x \in M$ outside a compact set an inequality:

$$
-b^{2} \leqslant \text { sectional curvature }(x) \leqslant-\frac{A}{\rho^{2}(x)}
$$

where $b, A$ are positive constants and $A>4$. Then $M$ admits a wealth of bounded harmonic functions. More precisely, the Dirichlet problem of the Laplacian of $M$ at infinity can be solved for any continuous boundary data on $S^{n-1}(\infty)$.

Theorem 2. Let $M$ be a Cartan-Hadamard manifold of dimension two. Suppose that $M$ satisfies for every $x \in M$ outside a compact set an inequality:

$$
-b^{2} \leqslant \text { sectional curvature }(x) \leqslant-\frac{A}{\rho^{2}(x)}
$$

where $b, A$ are positive constants and $A>2$. Then $M$ admits a wealth of bounded harmonic functions. More precisely, the Dirichlet problem of the Laplacian of $M$ at infinity can be solved for any continuous boundary data on $S^{1}(\infty)$. 
Remark1.1. To establish the solvability of the Dirichlet problem at infinity is a natural and beautiful way to show the existence of bounded harmonic functions on a CartanHadamard manifold. It is a standard method. We wonder if the restriction $A>4$ (when $n \geqslant 3$ ) and $A>2$ (when $n=2$ ) in Theorems 1 and 2 can be deleted or not. However, it is necessary in our arguments.

We shall first present some terminology, set up notation and recall some basic facts in Section 2, Theorems 1 and 2 will be proved in Section 3, and some further discussion will be given in Section 4 .

\section{Preliminaries}

Throughout this paper, unless otherwise stated,we always denote by $M$ an $n$ dimensional Cartan-Hadamard manifold, that is, a complete simply-connected Riemannian manifold of nonpositive sectional curvature. It is a well known fact about these manifolds that the exponential mapping at each point $x, \exp _{x}: T_{x} M \rightarrow M$, is a diffeomorphism. The sphere $S^{n-1}(\infty)$ at infinity of such a manifold $M$ is defined to be the set of asymptotic classes of geodesic rays in $M$. (Two rays $\gamma_{1}$ and $\gamma_{2}$ are asymptotic if $\operatorname{dist}\left(\gamma_{1}(t), \gamma_{2}(t)\right)$ is bounded for all $t \geqslant 0$.) If we choose a point $O \in M$ as the origin of the rays, then, from the viewpoint of geometry, $S^{n-1}(\infty)$ is the collection of rays emanating from point $O\left(=S_{O}^{n-1}(1) \subset T_{O} M\right)$ (see [1] or [2] for details).

There is a natural topology, called the cone topology on $\bar{M}$ (for details see [1]). Under this cone topology, $\bar{M}, M$ and $S^{n-1}(\infty)$ are homeomorphic to $\bar{B}(1) \subset R^{n}, B(1)$ and $S^{n-1}(1)$ respectively. This is the compactification of $M$. It should be pointed out that there is no natural differential structure on $S^{n-1}(\infty)$. But, if necessary, we use the above homeomorphism to identify $S^{n-1}(\infty)$ with $S^{n-1}(1) \subset R^{n}$.

Although our main object is to study Cartan-Hadamard manifolds, we have found it advantageous to work in the category of models (see [6]). The models not only provide a wealth of concrete manifolds with certain geometric features, but also allow us to compare the geometry of some kinds of non-symmetric manifolds with them. Let $R^{n}$ be a linear vector space of $n$ dimension endowed with a Riemannian metric $d s^{2}=d \rho^{2}+f^{2}(\rho) d \theta^{2}$, where $(\rho, \theta)$ are the usual polar coordinates of $R^{n}, f \in C^{\infty}(R)$ is a positive function with $f(0)=0, f^{\prime}(0)=1$, and $d \theta^{2}$ denotes the standard metric of $S^{n-1}(1)$. For this model $\widetilde{M}=\left(R^{n}, d s^{2}\right)$, by a direct calculation, we have [4]:

PROPOSITION 2.1. The Riemannian curvature tensor of the model $\widetilde{M}$ at point $(\rho, \theta)$ is expressed as follows:

$$
\widetilde{R}_{i j k l}=\left\{\begin{array}{l}
-\frac{f^{\prime \prime}}{f}\left(g_{i k} g_{j l}-g_{i l} g_{j k}\right), \quad \text { when at least one of } i, j, k, l=1 \\
\frac{1-\left(f^{\prime}\right)^{2}}{f^{2}}\left(g_{i k} g_{j l}-g_{i l} g_{j k}\right), \quad \text { when none of } i, j, k, l=1
\end{array}\right.
$$


where $g_{11}=1, g_{1 j}=g_{j 1}=0(j \neq 1), g_{i j}=\delta_{i j} f^{2}(\rho)(i, j \neq 1)$, and $\delta_{i j}$ is the Kronecker symbol.

Now we turn to the properties of harmonic functions on $M$. Let $\Delta$ and $\nabla$ denote the Laplacian and gradient of $M$ respectively. If $\operatorname{Ric}(M) \geqslant-b^{2}$ for some constant $b>0$, then we have the Harnack Principle: A sequence of harmonic functions $u$ on a domain $\Omega \subset M$ such that $|u| \leqslant K<\infty$ has a convergent subsequence which converges to a smooth harmonic function (see [2]). We also recall the well known maximum principle of the operator $\Delta$ : For any compact domain $\Omega \subset \subset M$ and harmonic function $u$ on $\Omega$, the maximum and minimum of $u$ in $\bar{\Omega}$ are achieved only at points on $\partial \Omega$. It is straightforward to verify that the maximum principle remains valid for any domain $\Omega \subset \bar{M}$ as well (also see to [2]).

\section{The Solvability of the Dirichlet Problem at Infinity}

Let $M$ be as in the Main Theorem, that is, $M$ satisfies the following inequality outside a compact set:

$$
-b^{2} \leqslant \text { sectional curvature }(x) \leqslant-\frac{A}{\rho^{2}(x)},
$$

where $A>4$. The crucial point is to give a geometric characterisation of the curvature restriction $\leqslant-\frac{A}{\rho^{2}(x)}$. This leads us to consider the models mentioned in Section 2. Take the model $\widetilde{M}=\left(R^{n}, d s^{2}\right)$ with $f(\rho)=\rho(1+\rho)^{1+\alpha}$ for some constant $\alpha>0$ which will be determined later. Because of Proposition. 2.1 in Section 2, by a direct calculation, we have

$$
\begin{aligned}
-\frac{f^{\prime \prime}}{f} & =-\frac{2(1+\alpha)}{\rho(1+\rho)}-\frac{\alpha(1+\alpha)}{(1+\rho)^{2}} \\
& \geqslant-\frac{2(1+\alpha)+\alpha(1+\alpha)}{\rho^{2}}
\end{aligned}
$$

and

$$
\begin{aligned}
\frac{1-\left(f^{\prime}\right)^{2}}{f^{2}} & =-\frac{1}{\rho^{2}}-\frac{2(1+\alpha)}{\rho(1+\rho)}-\frac{(1+\alpha)^{2}}{(1+\rho)^{2}}+\frac{1}{\rho^{2}(1+\rho)^{2+2 \alpha}} \\
& \geqslant-\frac{(2+\alpha)^{2}}{\rho^{2}} .
\end{aligned}
$$

From (3.2) and (3.3), we see that when $\alpha \leqslant 1$,

$$
\text { sectional curvature }(\rho, \theta) \text { of } \widetilde{M} \geqslant-\frac{(2+\alpha)^{2}}{\rho^{2}} \text {. }
$$


Hence when $n \geqslant 3$, for any given $A>4$ in (3.1), we may choose a suitable small $\alpha>0$ such that

$$
A>(2+\alpha)^{2}>4
$$

Since $M$ is diffeomorphic to $R^{n}$, we choose a fixed point $O \in M$ as the origin and let $\left\{(\rho, \theta) ; \theta \in S_{O}^{n-1}(1)\right\}$ be the normalised geodesic coordinates at $O$. Then, for the $\alpha$ chosen as in (3.5), the curvature condition (3.1) become

$$
\begin{aligned}
-b^{2} & \leqslant \text { sectional curvature }(\rho, \theta) \text { of } M \\
& \leqslant \text { sectional curvature }(\rho, \theta) \text { of } \widetilde{M}
\end{aligned}
$$

outside a compact set. This allows us to compare the geometry of $M$ with $\widetilde{M}$.

Remark 3.1. When $n=2$ from (3.2) for $A>2$ we can choose $\alpha>0$ small enough such that

$$
A>(2+\alpha)(1+\alpha)>2 .
$$

Then the curvature condition (3.1) with $A>2$ implies (3.6) for $\alpha$ chosen as above. It is easy to see that Lemma 3.1 is valid for such $\alpha$ when $n=2$.

REMARK 3.2. With the same argument as above, we can construct a lot of models $\left(R^{n}, d s^{2}=d \rho^{2}+\rho(1+\rho)^{1+\alpha} d \theta^{2}\right)$ satisfing the following inequality outside a compact set:

$$
-\frac{B}{\rho^{2}} \leqslant \text { sectional curvature }(x) \leqslant-\frac{A}{\rho^{2}}
$$

with $A>4$.

Lemma 3.1. Suppose $n \geqslant 3$. Let $x_{1}, x_{2} \in M$ with dist $\left(x_{1}, x_{2}\right) \leqslant 1$ and let $\theta$ be the angle of the geodesic rays from $O$ to $x_{1}$ and from $O$ to $x_{2}$. Then

$$
\theta \leqslant \frac{C}{(1+\rho(x))^{2+\alpha}} \quad\left(\text { when } \rho\left(x_{1}\right)\right. \text { is large enough) }
$$

where $C>0$ is a constant.

Proof: Without loss of generality, we assume that (3.1) or (3.6) holds outside a geodesic ball $B_{O}\left(R_{0}\right) \subset M$ for some $R_{0}<\infty$. Since $x_{1}, x_{2} \in M$ with dist $\left(x_{1}, x_{2}\right) \leqslant 1$, we see that, when $\rho\left(x_{1}\right)$ is sufficiently large, $x_{1}, x_{2}$ are located outside the ball $B_{O}\left(R_{0}\right)$. Let $y$ be the intersection point of the ray $O x_{1}$ and $\partial B_{O}\left(R_{0}\right)$. Obviously, we have

$$
\theta=\angle x_{2} O x_{1}<\angle x_{2} y x_{1},
$$

for $M$ is a Cartan-Hadamard manifold. 
We choose two points $\widetilde{x}_{1}, \widetilde{x}_{2} \in \widetilde{M}$ such that $\rho\left(\widetilde{x}_{1}\right)=\operatorname{dist}_{M}\left(y, x_{1}\right), \rho\left(\widetilde{x}_{2}\right)=$ $\operatorname{dist}_{M}\left(y, x_{2}\right)$ and $\operatorname{dist}_{\tilde{M}}\left(\widetilde{x}_{1}, \widetilde{x}_{2}\right)=\operatorname{dist}_{M}\left(x_{1}, x_{2}\right)$. From the geometric structure of $\widetilde{M}$, we see that

$$
\angle \tilde{x}_{2} O \tilde{x}_{1} \leqslant \frac{C}{\left(1+\rho\left(\tilde{x}_{1}\right)\right)^{2+\alpha}} \quad\left(\text { when } \rho\left(\tilde{x}_{1}\right)>>1\right)
$$

for some constant $C>0$. By using Toponogov's triangle comparison theorem, we have

$$
\angle x_{2} y x_{1} \leqslant \angle \tilde{x}_{2} O \tilde{x}_{1} \leqslant \frac{C}{\left(1+\rho\left(x_{1}\right)\right)^{2+\alpha}} .
$$

(Here we have used (3.6).) Combining (3.8) with (3.9), we get the desired inequality:

$$
\theta \leqslant \frac{C}{\left(1+\rho\left(x_{1}\right)\right)^{2+\alpha}}, \quad\left(\text { when } \rho\left(x_{1}\right)>>1\right)
$$

for some constant $C>0$. It is easy to see that we can restrict $\alpha<\frac{1}{R_{0}+1}$. This proves the lemma.

Now, we are in a position to prove Theorems 1 and 2.

Proof of Theorem 1: We fix a point $O \in M$ as the origin in $M$ and identify $S^{n-1}(\infty)$ with the collection of the geodesic rays emanating from $O$, that is, $S_{O}^{n-1}(1) \subset$ $T_{O} M$. It is well known that any continuous functions on $S^{n-1}(\infty)$ can be uniformly approximated by a sequence of Lipschitz functions on $S^{n-1}(\infty)=S_{O}^{n-1}(1)$. By using the Harnack Principle and maximum principle mentioned in Section 2, we see that if a harmonic sequence $\left\{u_{k} \in C^{\infty}(M) \cap C^{0}(\bar{M})\right\}$ converges uniformly on $S^{n-1}(\infty)$, then it converges uniformly on $\bar{M}$ to a harmonic function in the class $C^{\infty}(M) \cap C^{0}(M)$. Therefore, without loss of generality, we may assume that the boundary data $\varphi$ is a Lipschitz function on $S^{n-1}(\infty)$.

Since $M$ is a Cartan-Hadamard manifold and $\operatorname{expo}: T_{O} M \approx R^{n} \rightarrow M$ is a diffeomorphism, we let $\left\{(\rho, \theta) ; \theta \in S_{O}^{n-1}(1)\right\}$ be the normalised geodesic coordinates at $O \in M$ and $\varphi=\varphi(\theta), \theta \in S_{O}^{n-1}(1)=S^{n-1}(\infty)$. Extend $\varphi$ radially along the rays emanating from $O$, that is,

$$
\varphi(\rho, \theta)=\varphi(\theta), \quad \forall \rho>0 .
$$

The extended function is still denoted by $\varphi$, and is a bounded function on $M \backslash\{O\}$.

As in [2], we introduce the notation $\underset{B_{x}(1)}{o s c}=\sup _{y \in B_{x}(1)}|\varphi(y)-\varphi(x)|$ which is the amplitude of $\varphi$ on the geodesic ball $B_{x}(1)$ in $M$. 
The proof is divided into three steps:

Step 1: $\underset{B_{x}(1)}{\operatorname{osc}} \varphi=O\left(1+\rho(x)^{-(2+\alpha)}\right)$ when $\rho(x)>>1$, where $\rho(x)$ is the distance function from the origin $O$ to $x$.

In fact, since $\varphi$ is Lipschitz, that is, $\forall y \in B_{x}(1)$

$$
|\varphi(y)-\varphi(x)|=\left|\varphi\left(\theta_{y}\right)-\varphi\left(\theta_{x}\right)\right| \leqslant C\left|\theta_{y}-\theta_{x}\right|
$$

where $\theta_{y}$ and $\theta_{x}$ denote the sphere coordinates of $y$ and $x$ respectively, $\left|\theta_{y}-\theta_{x}\right|$ is the angle between the rays $O x$ and $O y$ at the origin and $C$ is a constant. And from lemma 3.1 , we have

$$
\left|\theta_{y}-\theta_{x}\right| \leqslant \frac{C}{(1+\rho(x))^{2+\alpha}}
$$

for some $\alpha>0$. Therefore, we get

$$
\underset{B_{x}(1)}{\operatorname{osc}} \varphi \leqslant \frac{C}{(1+\rho(x))^{2+\alpha}} .
$$

Step 2: There is a $C^{2}$ function $\tilde{\varphi}$ on $M$ with boundary data $\varphi$ on $S^{n-1}(\infty)$ and such that

$$
|\Delta \tilde{\varphi}|=O\left((1+\rho(x))^{-(2+\alpha)}\right)
$$

In fact, we define the average function $\tilde{\varphi}$ of $\varphi$, with respect to a function $\chi$, by

$$
\tilde{\varphi}=\frac{\int_{M} \chi\left(\rho_{x}^{2}(y)\right) \varphi(y) d y}{\int_{M} \chi\left(\rho_{z}^{2}(y)\right) d y}
$$

where $\chi: R \rightarrow R$ is a $C^{2}$ function satisfying $\sup \chi \subset[-1,1]$ and $\chi=1$ on $\left[0, \frac{1}{2}\right]$, $0 \leqslant \chi \leqslant 1$. Obviously, $\tilde{\varphi}$ is a $C^{2}$ function on $M$. Under the assumption $-b^{2} \leqslant$ sectional curvature of $M \leqslant 0$, with the same argument as in [2], we can get

$$
\begin{aligned}
& |\tilde{\varphi}(x)-\varphi(x)| \leqslant \underset{B_{x}(1)}{\operatorname{osc}} \varphi \\
& |\Delta \tilde{\varphi}(x)| \leqslant C \underset{B_{x}(1)}{\operatorname{osc} \varphi}
\end{aligned}
$$

where $C>0$ is a constant. Therefore, Step 1 leads to

$$
|\Delta \tilde{\varphi}(x)|=O\left((1+\rho(x))^{-(2+\alpha)}\right) .
$$

It is easy to see that the $\tilde{\varphi}$ is the desired function. 
Step 3: Consider a $C^{\infty}$ function $g(x)=\frac{1}{(1+\rho(x))^{\delta}}$ on $M \backslash\{O\}(\delta>0)$. It is easy to see that

$$
\Delta g=-\frac{\delta \Delta \rho(x)}{(1+\rho(x))^{1+\delta}}+\frac{\delta(1+\delta)|\nabla \rho(x)|^{2}}{(1+\rho(x))^{2+\delta}}
$$

As in Lemma 3.1, we assume that (3.4) or (3.6) holds outside a geodesic ball $B_{o}\left(R_{0}\right)$ for some $R_{0}<+\infty$. Since $M$ is an $n$ dimensional Cartan-Hadamard manifold, from the Hessian comparison theorem, we know that

$$
\Delta \rho(x) \geqslant \frac{n-1}{\rho(x)} \quad \rho(x)>0
$$

on $M \backslash\{O\}$. Thus, from (3.13), we have

$$
\begin{aligned}
\Delta g & \leqslant \frac{\delta(1+\delta)}{(1+\rho(x))^{2+\delta}}-\frac{(n-1) \delta}{(1+\rho(x))^{1+\delta} \rho(x)} \\
& =-\frac{\delta}{(1+\rho(x))^{2+\delta}}\left[n-2+\frac{n-1}{\rho(x)}\right]<0(\text { when } \rho(x)>0) .
\end{aligned}
$$

Now, choose a fixed $\delta \leqslant \alpha$ such that (3.14) is valid. Since $\Delta \tilde{\varphi}=O\left((1+\rho(x))^{-(2+\alpha)}\right)$ from Step 2, when the constant $C$ is sufficient large, we get

$$
\Delta(C g) \leqslant-|\Delta \tilde{\varphi}|,
$$

that is, $\Delta(\tilde{\varphi}+C g) \leqslant 0$ and $\Delta(\tilde{\varphi}-C g) \geqslant 0$.

So $\widetilde{\varphi}+C g$ is a superharmonic function on $M$ with boundary value $\varphi$ on $S^{n-1}(\infty)$ and $\widetilde{\varphi}-C g$ is a subharmonic function on $M$ with boundary value $\varphi$. The functions $\tilde{\varphi}+C g$ and $\tilde{\varphi}-C g$ may be used as barrier functions to solve the Dirichlet problem on $S^{n-1}(\infty)$ via the Perron method. Therefore, there is unique harmonic function $u \in C^{\infty}(M) \cap C^{0}(M)$ satisfing $\widetilde{\varphi}-C g \leqslant u \leqslant \widetilde{\varphi}+C g$ and achieving the desired boundary value $\varphi$ on $S^{n-1}(\infty)$. The latter assertion is from the following fact:

$$
\begin{aligned}
|u-\varphi|(x) & \leqslant|u-\tilde{\varphi}+\tilde{\varphi}-\varphi|(x) \\
& \leqslant C g(x)+|\tilde{\varphi}-\varphi|(x) \\
& \leqslant C \frac{1}{(1+\rho(x))^{\delta}}+O\left((1+\rho(x))^{-(2+\alpha)}\right) \\
& \rightarrow 0 \quad(\text { as } \rho(x) \rightarrow \infty) .
\end{aligned}
$$

This completes the proof of the Theorem 1 . 
Proof of Theorem 2: Most of the details are the same as in the proof of Theorem 1. By Remark 3.1 the difference lies in Step 3 of the proof of Theorem 1. We consider the $C^{\infty}$ function $g(x)=\frac{1}{(1+\rho(x))^{\delta}}$ on $M \backslash\{O\}(\delta>0)$.

As in Lemma 3.1, we assume that (3.4) or (3.6) holds outside a geodesic ball $B_{o}\left(R_{0}\right)$ for some $R_{0}<+\infty$ Since $M$ is an 2 dimensional Cartan-Hadamard manifold, from the Hessian comparison theorem, we know that

$$
\Delta \rho(x) \geqslant \begin{cases}\frac{1}{\rho(x)} & \rho(x)>0 \\ \frac{2}{1+\rho(x)} & \rho(x)>R_{0}\end{cases}
$$

on $M \backslash\{O\}$. Thus from (3.13) we have

$$
\begin{gathered}
\Delta g \leqslant \frac{\delta(1+\delta)}{(1+\rho(x))^{2+\delta}}-\frac{2 \delta}{(1+\rho(x))^{2+\delta}} \\
=-\frac{\delta(4-(3+\delta))}{(1+\rho(x))^{2+\delta}}<0\left(\text { when } \rho(x)>R_{0}+1\right), \\
\Delta g \leqslant \frac{\delta}{(1+\rho(x))^{2+6}}\left(-\frac{1+\rho(x)}{\rho(x)}+1+\delta\right) \\
=\frac{\delta}{(1+\rho(x))^{2+\delta}}\left(-\frac{1}{\rho(x)}+\delta\right)<0\left(\text { when } \rho(x) \leqslant 1+R_{0}\right)
\end{gathered}
$$

when $\delta<\frac{1}{R_{0}+1}$ is small. Now, choose a fixed $\delta \leqslant \alpha$ such that (3.15) is valid. Since $\Delta \widetilde{\varphi}=O\left((1+\rho(x))^{-(2+\alpha)}\right)$ from Step 2 , when the constant $C$ is sufficiently large, we get

$$
\Delta(C g) \leqslant-|\Delta \widetilde{\varphi}|,
$$

that is, $\Delta(\tilde{\varphi}+C g) \leqslant 0$ and $\Delta(\tilde{\varphi}-C g) \geqslant 0$. By the same method as in the proof of Theorem 1 we can see that the conclusion of Theorem 2 will hold.

REMARK 3.3. It is easy to see that the result due to Anderson and Sullivan (see [1] and [8]) is a special case of our Main Theorem. From Remark 3.1, we also see that there are lots of Cartan-Hadamard manifolds satisfying the conditions in our theorem but not satisfying the negative sectional curvature pinching conditions. Based on this Main Theorem, we can study the harmonic maps that map $S^{n-1}(\infty)$ of such a manifold into a bounded convex ball of another manifold. We shall discuss it in another paper.

The solvability of the Dirichlet problem leads naturally to the harmonic measure and the Poisson kernel as Anderson and Schoen studied in [2]. Similarly, we have:

Proposition 3.2. Let $M$ be as in the Main Theorem. Then, for almost all $Q \in S^{n-1}(\infty)$, the Poisson kernel $K(x, Q)$ (see [2]) at infinity exists. 


\section{Further Discussions}

As pointed out in Remark 1.1 in Section 1, we wonder if the restriction $A>4$ $(n \geqslant 3)$ or $A>2(n=2)$ is necessary to guarantee the existence of bounded harmonic functions on the manifold. If $M$ is a 2 -dimensional simply connected complete Riemannian manifold with Gaussian curvature $K(x) \leqslant-\frac{A}{1+r^{2}(x)}$ and rotational symmetry about a point, then we claim that $M$ has enough bounded harmonic functions. In fact this is a direct corollary of a theorem obtained by Milnor in [7]. For omitting the restriction on $A$, we have the following interesting results which are straightforward from Theorem 1 and 2.

Theorem 4.1. Let $M$ be a Cartan-Hadamard manifold of dimension $n$ ( $n \geqslant$ 2). Suppoise that $M$ satisfies for every $x \in M$ outside a compact set an inequality:

$$
-b^{2} \leqslant \text { sectional curvature }(x) \leqslant-\frac{A}{\rho^{2-\varepsilon}(x)}
$$

where $\ddot{b}, A$, and $\varepsilon$ are positive constants. Then $M$ admits a wealth of bounded harmonic functions. More precisely, the Dirichlet problem of the Laplacian of $M$ at infinity can be solved for any continuous boundary data on $S^{n-1}(\infty)$.

Proof: We only need to check that $M$ satisfies for large $\rho(x)$

$$
-b^{2} \leqslant \text { sectional curvature at } x \leqslant-\frac{B}{\rho^{2}(x)}
$$

for some fixed constant $B>4$. In fact for

$$
-\frac{A}{\rho^{2-\varepsilon}(x)}=-\frac{A \rho^{e}(x)}{\rho^{2}(x)}
$$

when $\rho(x)$ is sufficiently large we can obtain

$$
A \rho^{\varepsilon}(x)>B>4
$$

for some fixed constant $B$. By applying Theorems 1 and 2 in Section 1 we prove the theorem.

COROLLARY 4.1. If $M$ satisfies the following inequality outside a compact set:

$$
-b^{2} \leqslant \text { sectional curvature at } x \leqslant-\frac{C}{\rho(x)}
$$

for some positive constant $C$ then $M$ admits enough bounded harmonic functions. 


\section{REFERENCES}

[1] M.T. Anderson, 'The Dirichlet problem at infinity for manifold of negative curvature', $J$. Differential Geom. 18 (1983), 701-721.

[2] M.T. Anderson and R. Schoen, 'Positive harmonic function on complete manifolds of negative curvature', Ann. of Math 121 (1985), 420-461.

[3] W. Ballmann, 'Dirichlet problem at infinity for manifold of nonpositive curvature', Form. Math. 1 (1990), 201-213.

[4] J.C. Chen and J.Y. Li, 'A remark on eigenvalues', Chinese Sci. Bull. 9 (1989), 655-658.

[5] Q. Ding, 'The Dirichlet problem at infinity for manifolds of nonpositive curvature', in Differential geometry, (C.H. Gu, H.S. Hu, and Y.L. Xin, Editors) (World Sci. Publ. Co., Hong Kong, 1993), pp. 49-58.

[6] R. Greene and $\mathrm{H}$. Wu, Function theory on manifolds which possess a pole, Lecture Notes in Mathematics 699 (Spring-Verlag, Berlin, Heidelberg, New York, 1979).

[7] J. Milnor, 'On deciding whether a surface is parabolic or hyperbolic', Amer. Math. Monthly 84 (1977), 43-46.

[8] D. Sullivan, 'The Dirichlet problem at infinity for negative curved manifold', J. Differential Geom. 18 (1983), 723-732.

[9] S.T. Yau, 'Harmonic functions on complete Riemannian manifold', Comm. Pure Appl. Math. 28 (1975), 201-228.

[10] S.T. Yau, 'Some function-theoretic properties of complete Riemannian manifolds and their applications to geometry', Indiana Univ. Math. J. 25 (1976), 659-670.

Institute of Mathematics

Fudan University

Shanghai 200433

China
Department of Mathematics

Shandong University

Jinan, Shandong 250100

China 